\title{
Highly efficient and tunable selective absorber design based on metallic nanoparticles in a graded index dielectric
}

\author{
Rafif Hamam* and Ali J. Sabbah \\ Department of Mathematics and Physics, The International University of Beirut, Beirut, Lebanon
}

Received: 12 October 2018 / Accepted: 21 January 2019

\begin{abstract}
We propose a novel solar selective absorber design based on transverse localized surface plasmon resonances of infinite metallic nanorods embedded in a graded index dielectric slab. The physics principles on which the design is based are explained, and decent results are obtained by numerical simulations; solar absorptance values exceeding 0.99 are reached together with a near-zero infrared emittance. The proposed structure design offers a flexible tunability of thermal emission, and this spectral control over thermal emission promises advances not only in solar energy harvesting efficiency, but also in sensing, camouflage, and other thermal management applications.
\end{abstract}

Keywords: Surface plasmon resonance / nanorods / graded index material / solar selective absorber / thermal emission design

\section{Introduction}

In the last few decades, light trapping has been recognized as a critical factor in enhancing the efficiency (and possibly reducing the cost) of solar energy harvesting devices [1-3], such as photovoltaic (PV) cells [4], thermophotovoltaic (TPV) converters [5,6], solar thermoelectric generators $[7,8]$, concentrated solar thermal power plants (CSP) [9], or even solar water heaters. A common objective sought after while attempting to improve the efficiency of solar energy conversion technologies is to maximize the absorption of the solar spectrum part that can be eventually converted into electricity, while minimizing the infrared (IR) thermal radiation loss. In single-junction $\mathrm{PV}$ devices, only the portion of the solar spectrum with photon energies exceeding the semiconductor energy gap $E_{g}$ is useful. However, solar thermal conversion devices extend this useful range to a significantly wider portion of the solar spectrum ranging from the ultraviolet (UV) at around $1000 \mathrm{THz}$ (corresponding to $0.3 \mu \mathrm{m}$ ) down to the IR at $150 \mathrm{THz}$ (corresponding to $2 \mu \mathrm{m}$ ), as illustrated by the shaded area of Figure 1a.

This promising advantage in solar thermal conversion [11-13] requires the design of frequency selective solar absorbers that efficiently convert sunlight to heat. The generated heat could be subsequently converted to electricity by various possible technologies (TPVs, heat engines, thermoelectrics, ....) or even used directly as in

\footnotetext{
* e-mail: rhamam.biu.edu@gmail.com
}

solar water heaters. As a consequence of collecting a significant portion of the incident solar radiation, the absorber's temperature reaches high values (typically around $1000-2000 \mathrm{~K}$ in TPV applications, and around 700-1200 K in CSPs). Hence, the absorber will lose energy by thermal emission at IR frequencies; according to Planck's law of radiation, a blackbody at $1000 \mathrm{~K}$ would have a peak radiation at a wavelength around $3 \mu \mathrm{m}$ corresponding to $100 \mathrm{THz}$, as shown in Figure 1a. Hence, an ideal selective absorber, operating at a high temperature $T_{h}$, needs to have an absorptance of 1 over the entire solar spectrum down to a cutoff frequency $f_{c}$, and an emittance of zero at IR frequencies below $f_{c}$. This cutoff frequency is determined by the absorber's specific temperature $T_{h}$ so as light trapping is optimized; maximum solar energy needs to be absorbed with minimum IR energy thermally reradiated. For example, in the blackbody spectrum shown in Figure 1a for a tempeature $T_{h}=1000 \mathrm{~K}$, although radiated energy extends up to a frequency slightly above $300 \mathrm{THz}$, it remains significant only up to around $200 \mathrm{THz}$. However, in the AM1.5 spectrum [10], also shown in the same figure, solar radiation starts to get significant from frequencies around $150 \mathrm{THz}$, peaks at $600 \mathrm{THz}$, and extends up to $1000 \mathrm{THz}$. So, for an absorber at $1000 \mathrm{~K}$, one can compromise absorbing the small amount of incoming solar energy at frequencies below $150 \mathrm{THz}$, for the sake of suppressing the significant IR radiation loss at those frequencies (since, under thermal equilibrium, absorptance equals emittance at each frequency according to Kirchhoff's law of thermal radiation). Hence, the cutoff frequency 


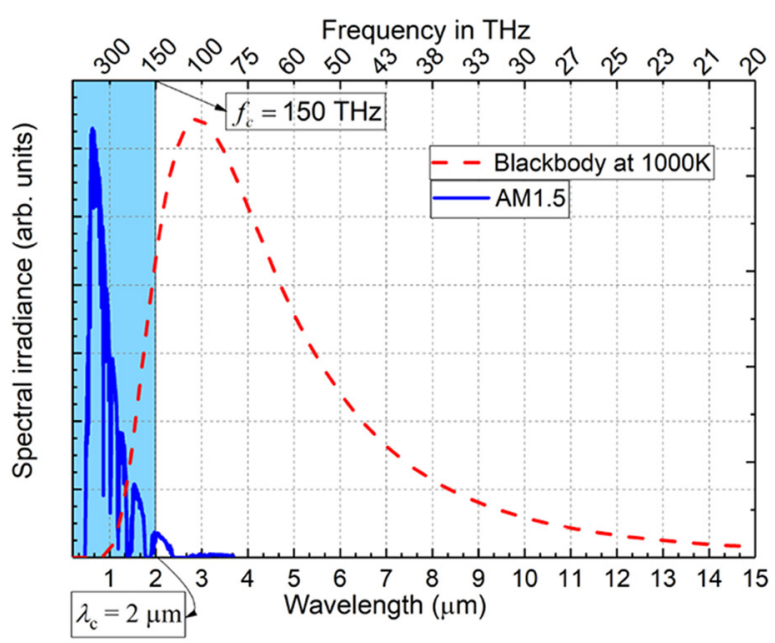

(a)

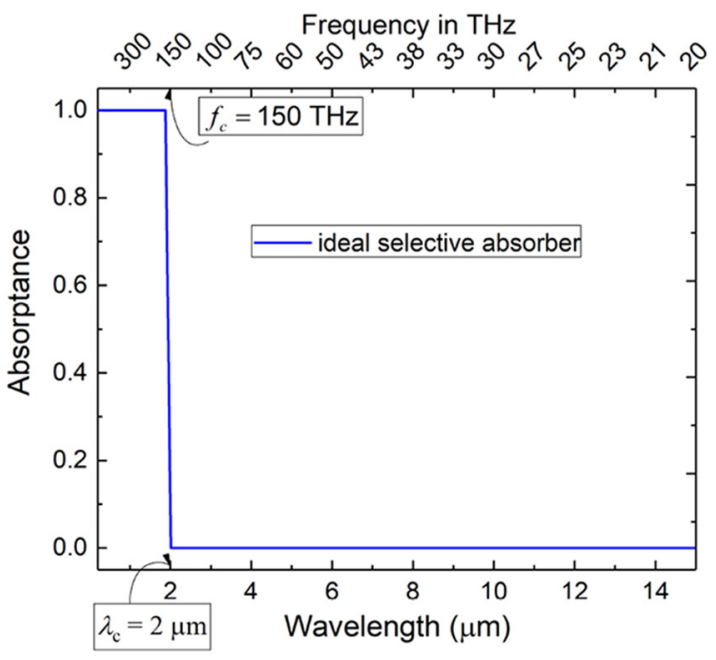

(b)

Fig. 1. (a) AM1.5 spectrum (solar spectrum at sea level for a solar zenith angle of $48.2^{\circ}$ ) [10], and blackbody spectrum (in dashed red) corresponding to $1000 \mathrm{~K}$. (b) Desired absorptance spectrum in an ideal absorber case.

can be taken to be $f_{c}=150 \mathrm{THz}\left(\lambda_{c}=2 \mu \mathrm{m}\right)$ for an ideal absorber at $T_{h}=1000 \mathrm{~K}$. In Figure $1 \mathrm{~b}$, we show the absorptance of an ideal selective absorber at $1000 \mathrm{~K}$. At $T_{h}$ higher than $1000 \mathrm{~K}$, the peak of IR thermal radiation shifts to larger frequencies, so $f_{c}$ needs to be larger than $150 \mathrm{THz}$. One should note, however, that the high absorber temperatures involved in solar thermal conversion impose a practical challenge; the metallic and dielectric components to be used in the design of the selective absorber need to be thermally stable and have a high melting point, which limits the material choices.

While ideal selective absorbers have not been realized, practical ones have reached close enough, with a solar absorptance $A_{\text {solar }}$ near 0.95 and IR emittance $\varepsilon_{\mathrm{IR}}$ near 0.05 . Various designs have been proposed and implemented [14-18], and they were classified into six categories [19]. The best performing absorbers that have been experimentally reported so far are mostly of the multilayer, texturedsurface, and cermet types, all of which include metallic components [1]. The multilayer absorber (also called multilayer interference stacks) type is based on multiple reflections, whereas the textured-surface type relies on controlling surface roughness, so that the surface appears smooth to long wavelength IR radiation, while sunlight at shorter wavelengths experiences the fine details of the properly textured surface and gets trapped and absorbed. As for the cermet absorber, it consists of an antireflection coating, absorbing cermet layer(s) made up of metallic nanoparticles (of a graded concentration) embedded in a dielectric, and a bottom metal that reflects the IR [19]. The broadband solar absorption in the cermet layer(s) is achieved by tuning the nanoparticles' size, shape, coating, orientation, concentration gradient (gradient in the metal volume fraction), etc.

In this article, we propose a highly efficient singlelayer frequency selective absorber design based on localized surface plasmon resonances (SPRs) of metallic nanoparticles embedded in a graded-index dielectric slab. While localized SPRs enable significant absorption over a narrow frequency range around a frequency $f_{s p}$, the use of the graded index dielectric will be shown to extend this significant absorption over the entire solar spectrum down to $f_{c}=150 \mathrm{THz}$, the desired cutoff frequency at $T_{h}=1000 \mathrm{~K}$. This result will be validated by numerical simulations for transverse magnetic (TM) polarized incident plane wave light in the case of cylinder-shaped nanowires, and it will be noted how the design can be easily extended to work for the transverse electric (TE) polarization as well. While the best performing experimentally reported absorbers either require muliple layers (as in the multilayer type), or a fine tuning of the nanoparticles' concentration, size, orientation, etc. (as in the cermet type), or even properly orienting the textured surface's grooves and details (as in the textured surface type), our proposed selective absorber consists of a single layer without the need of any surface texturing. Although our design is based on nanoparticles resonance, like the cermet type absorbers, the main difference is that, instead of varying the nanoparticles' parameters (size, orientation, shape, concentration, etc.) in order to achieve broadband solar absorptance, we vary the dielectric environment of the nanoparticles by embedding them in a graded index slab.

The article is structured as follows: in Section 2, we review some facts about the localized transverse SPR of a single metallic cylinder of infinite length, and we discuss how the frequency of this SPR can be significantly varied by modifying the dielectric environment surrounding the cylinder. We then explore how a graded index dielectric can expand the frequency range of SPR absorption, and at the same time reduce reflection. In Section 3, we propose a wellrationalized design for a frequency selective absorber, and we explain our choices for the specific metal materials and also for the cylinder radii used. Then, we study numerically the performance of the proposed structure by doing 


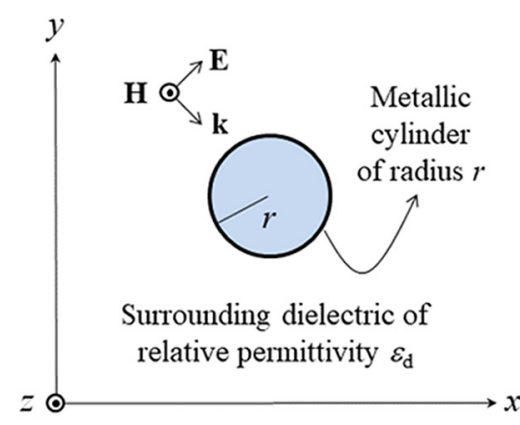

(a)

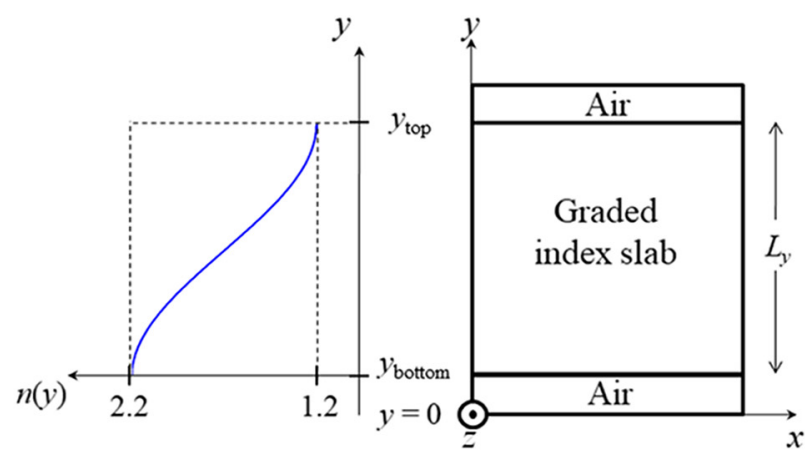

(b)

Fig. 2. Schematic diagram of (a) a metallic rod surrounded by a dielectric of relative permittivity $\varepsilon_{d}=n^{2}$, and (b) a slab sandwiched by air on both sides, and having the graded index profile $n(y)$ shown at the left.

numerical simulations for reflectance, transmittance, and absorptance spectra both at normal and oblique incidences. Finally, in Section 4, we illustrate the flexible tunability advantage of our proposed design by clarifying how high absorption in a certain frequency interval can be mapped to specific rod locations. In fact, by keeping/removing these specific rods (at the design stage), one can, in principle, enhance/suppress thermal emission in this frequency interval, which is a promising step in the control of thermal emission.

\section{Concept of the absorber design}

Consider an infinitely long metallic cylinder of nanosize radius $r$ embedded in a dielectric medium of dielectric constant $\varepsilon_{d}$. The cylinder's axis is along $z$, and its dielectric function $\varepsilon_{m}$ has the Drude form:

$$
\varepsilon_{m}(\omega)=\varepsilon_{\infty}-\frac{\omega_{p}^{2}}{\omega^{2}+i \omega \gamma}
$$

where $\omega_{p}$ is the metal's plasma frequency, $\gamma$ is the bulk metal damping constant, and $\varepsilon_{\infty}$ is the high frequency permittivity. At the moment, we restrict our attention to TM light waves of angular frequency $\omega_{\text {inc }}=2 \pi f_{\text {inc }}$ incident on this cylinder. For such TM waves, the magnetic field is along the $z$ axis, while the electric field is in the $x y$ plane (perpendicular to the cylinder's axis), as shown in Figure $2 \mathrm{a}$. When the incident light frequency $f_{\text {inc }}$ is close enough to a certain natural frequency $f_{s p}$, light drives the conduction electrons of the metallic cylinder into resonant oscillations damped by the positive ions and by the cylinder's surface. Such oscillations entail a reduction (extinction) of the incident light's energy at $f_{s p}$, and hence a resonant light absorption due to the excitation of a SP oscillation [20]. For an infinitely long metallic nanocylinder, the transverse SPR angular frequency $\omega_{s p}\left(=2 \pi f_{s p}\right)$ is given by the Frohlich condition [21] $\operatorname{Re}\left(\varepsilon_{m}\right)=-\varepsilon_{d}$, which, upon neglecting the dielectric material's dispersion, results in

$$
\omega_{s p}=\frac{\omega_{p}}{\sqrt{\varepsilon_{\infty}+\varepsilon_{d}}} .
$$

As we can see from equation (2), the SPR frequency is determined by the metal material (through $\omega_{p}$ and $\varepsilon_{\infty}$ ) and also by the dielectric environment (through $\varepsilon_{d}$ ). Consequently, identical metallic nanorods placed in different dielectric environments will exhibit diferent values for $\omega_{s p}$. For instance, an infinite metallic nanowire (having $\varepsilon_{\infty}=1$ ) placed in a medium of refractive index $n=1.2$ will exhibit a SPR at:

$$
\omega_{s p}=\frac{\omega_{p}}{\sqrt{1+1.2^{2}}}=0.65 \omega_{p}
$$

while the same nanowire placed in a medium of refractive index $n=2.2$ will have:

$$
\omega_{s p}=\frac{\omega_{p}}{\sqrt{1+2.2^{2}}}=0.41 \omega_{p}
$$

Therefore, by dispersing numerous identical rods in a dielectric slab having a graded index profile varying continuously from $n=1.2$ to $n=2.2$, the rods at the various different positions in the slab will experience different dielectric environments, and will thus exhibit a "continuum" of surface plasmon angular frequencies ranging from $0.41 \omega_{p}$ to $0.65 \omega_{p}$. In Figure $2 \mathrm{~b}$, we show a cross section of a graded index dielectric slab [22] having a sinusoidally varying profile that starts from $n_{\text {top }}=1.2$ at the slab's top interface with air, and reaches $n_{\text {bottom }}=2.2$ at the slab's bottom interface with air. While at this stage, the slab's thickness $L_{y}$ seems to be arbitrary, we will clarify, in Section 3, our choice for the specific $L_{y}$ values used in the design. The slab's refractive index profile is given by

$$
n(y)=1.7+0.5 \times \cos \left(\frac{\pi}{L_{y}}\left(y-y_{\text {bottom }}\right)\right) .
$$

Such a graded index can be realized, for example, by using porous silicon (pSi), which is produced by electrochemical etching of a silicon ( $\mathrm{Si}$ ) wafer [23], and in which the gradual variation in the refractive index is achieved by a continuous change in porosity [24]. While the index gradient minimizes reflection at the top air-slab interface, the slab's constituent materials need to be thermally stable at particular high temperature $T_{h}$ of interest. In fact, the 
melting point of pSi depends on its porosity, but it is known to vary between $900^{\circ} \mathrm{C}$ and $1414^{\circ} \mathrm{C}$ [25] (corresponding to $1173-1687 \mathrm{~K}$ ). So, although the value of $1000 \mathrm{~K}$ used for $T_{h}$ falls below the melting point of pSi, careful investigations are needed regarding the slab's thermal, chemical, and mechanical stability at such temperatures [26], noting that some method [27] has been reported on how to minimize thermal effects in pSi up to $700^{\circ} \mathrm{C}$ (for $50 \%-70 \%$ porosity). Also, the specific value of $1000 \mathrm{~K}$ for $T_{h}$ was just taken as an example, and our design can be used at lower $T_{h}$ values (which would correspond to lower $f_{c}$ ).

\section{Design and performance of the proposed selective absorber}

To verify and implement the above-mentioned design concept for broadband absorption, we consider infinitely long metallic nanorods each of radius $r=14 \mathrm{~nm}$, embedded in a section of the graded index dielectric slab of Figure $2 b$. The rods are separated by a horizontal interparticle distance $a_{x}=600 \mathrm{~nm}$ and by a vertical interparticle distance $a_{y}=250 \mathrm{~nm}$. Such large separations $\left(a_{x} \gg r\right.$ and $\left.a_{y} \gg r\right)$ limit interparticle interactions and are needed for sharper SPRs. As shown in Figure 3a, three columns and nineteen rows of nanorods are used, with each column shifted vertically by $a_{y} / 3$ from the next to ensure that the rods sense a "continuum" of values for the surrounding refractive index in the graded index slab. With our choice for the number of rods per column (19), their vertical separation $a_{y}$ of $250 \mathrm{~nm}$, and with the vertical shift of $a_{y} / 3$ between adjacent columns, the required slab thickness needs to be around $20.5 a_{y}$, which corresponds to $L_{y}=5.12 \mu \mathrm{m}$.

Rhodium ( $\mathrm{Rh})$ is used as the nanorods' metal material, given its high melting point $(2237 \mathrm{~K})$, and also given its low plasma frequency $\omega_{p}^{\mathrm{Rh}}=3.8 \mathrm{eV}[28,29]$. (A low $\omega_{p}$ metal is needed in order to ensure that $\left[0.41 \omega_{p}, 0.65 \omega_{p}\right]$ is within the peak portion of the AM1.5 solar spectrum). The damping parameter $\gamma_{\mathrm{Rh}}$ is corrected from its bulk value by adding the term $v_{F} / 2 r$ in order to account for surface collisions [30]. The Fermi speed $v_{F}$ is taken to be $1.2 \times 10^{6} \mathrm{~m} / \mathrm{s}$ (within the range $0.75-2.25 \times 10^{6} \mathrm{~m} / \mathrm{s}$ of Fermi speed values tabulated in the literature [31]) and $\gamma_{\mathrm{Rh}}$ is taken $0.1 \mathrm{eV}$. Resonant absorption is expected for $0.41 \omega_{p}^{\mathrm{Rh}}<\omega_{\text {inc }}<$ $0.65 \omega_{p}^{\mathrm{Rh}}$, corresponding to

$$
380 \mathrm{THz}<f_{\text {inc }}<580 \mathrm{THz} .
$$

To validate this prediction, we perform numerical simulations in which the finite element method (locally available software) is used to solve Maxwell's equations in frequency-domain, with a TM polarized plane wave light of variable frequency $f_{\text {inc }} \in[25 \mathrm{THz}, 1000 \mathrm{THz}]$ being normally incident onto the top side of the slab from a distance of $800 \mathrm{~nm}$. Periodic boundary conditions are imposed at the left and right sides of the computational domain: $x=0$ and $x=L_{x}=3 a_{x}$. Reflectance $(R)$ and transmittance $(T)$ spectra are calculated, and then the absorptance $(A)$ spectrum is deduced by using $A=1-R-T$. The result is shown in Figure $3 \mathrm{~b}$, and is indeed consistent with the predictions of equation (6).
Although the structure proposed in Figure 3a exhibits broadband absorption over $f_{\text {inc }} \in[380 \mathrm{THz}, 580 \mathrm{THz}]$, it is still far from serving as an efficient solar thermal absorber. The objective is to additionally ensure high absorptance over [580 THz, $1000 \mathrm{THz}$ ] and also over [200 THz, $380 \mathrm{THz}$ ], in addition to high reflectance for $f_{\text {inc }}<150 \mathrm{THz}$ (corresponding to the cutoff wavelength $\lambda_{c}>2 \mu \mathrm{m}$ ).

In order to broaden the resonant absorption frequency range as just mentioned, we propose using two additional metal materials: one with $\omega_{p}>\omega_{p}^{\mathrm{Rh}}$ and such that $0.65 \omega_{p} \simeq 1000 \mathrm{THz}$, and another with $\omega_{p}<\omega_{p}^{\mathrm{Rh}}$ and such that $0.41 \omega_{p} \simeq 150 \mathrm{THz}$. Metal candidates that are closest to satisfying these criteria and having high melting points are: tungsten $(\mathrm{W})$ with $\omega_{p}^{W}=6.4 \mathrm{eV}$ [28] (in this case $0.65 \omega_{p}^{W}$ corresponds to $1005 \mathrm{THz}$ ), and titanium nitride (TiN) having $\omega_{p}^{\mathrm{TiN}}=2.65 \mathrm{eV}, \gamma_{\text {bulk }}^{\mathrm{TiN}}=0.13 \mathrm{eV}$, and $\varepsilon_{\infty}=2$ [32]. In this latter case, equation (2) yields $\omega_{s p}=0.38 \omega_{p}^{\mathrm{TiN}}$ in a dielectric medium of index 2.2 , and this corresponds to $243 \mathrm{THz}$.

We first replace the Rh rods of Figure $3 \mathrm{a}$ by tungsten rods, each of radius $r_{\mathrm{W}}=8 \mathrm{~nm}$, and accordingly, we modify the bulk damping parameter $\gamma$ (taken $0.1 \mathrm{eV}$ ) by adding $v_{F} / 2 r_{\mathrm{W}}$ to it. In this case, the numerically simulated $R, T$, and $A$ spectra are shown in Figure $3 \mathrm{c}$, and apart from some broadening effect and discretization errors, they are consistent with the predicted interval $\left[0.41 \omega_{p}^{\mathrm{W}}, 0.65 \omega_{p}^{\mathrm{W}}\right]$ corresponding to $[620 \mathrm{THz}, 1000 \mathrm{THz}]$.

In Figure 3d, we show the $R, T$, and $A$ spectra in the case where the rods are made out of TiN and each has a radius of $24 \mathrm{~nm}$. These TiN rods embedded in the graded index slab are expected to enable resonant absorption over $\left[0.38 \omega_{p}^{\mathrm{TiN}}, 0.54 \omega_{p}^{\mathrm{TiN}}\right]$ corresponding to $[243 \mathrm{THz}, 346 \mathrm{THz}]$, which is again in agreement with the absorption band of Figure 3d.

Therefore, the structure that we propose in this article as an efficient frequency selective absorber consists of a graded index dielectric slab in which three types of metal nanowires are embedded: TiN nanowires each of radius $24 \mathrm{~nm}$, Rh nanowires each of radius $14 \mathrm{~nm}$, and $\mathrm{W}$ nanowires each of radius $8 \mathrm{~nm}$. The TiN nanowires ensure absorption over $[243 \mathrm{THz}, 346 \mathrm{THz}]$, while the Rh nanowires result in resonant absorption in the interval $[380 \mathrm{THz}$, $580 \mathrm{THz}]$, and the $\mathrm{W}$ nanowires give resonant absorption over $[620 \mathrm{THz}, 1000 \mathrm{THz}]$. Given the frequency gaps [346 THz, $380 \mathrm{THz}]$ and $[580 \mathrm{THz}, 620 \mathrm{THz}]$ between these absorption intervals, and given that interparticle interactions have a broadening effect, we reduce the interparticle spacings $a_{x}$ and $a_{y}$ down to $80 \mathrm{~nm}$ and $130 \mathrm{~nm}$, respectively, so as the gaps get bridged, and the absorption spectrum of the proposed structure is closest to the ideal target of Figure $1 \mathrm{~b}$ with $f_{c} \simeq 150 \mathrm{THz}$. In Figure $4 \mathrm{a}$, we show the geometry for this proposed frequency selective absorber, and in Figure 4b, we show the numerically simulated $R, T$, and $A$ spectra for normally incident TM polarized light.

One can see how our proposed structure exhibits an almost ideal performance with values of $R$ nearly 1 at low IR frequencies, and values of $A$ aproaching $0.99-1$ for a wide range of frequencies in the interval $[200 \mathrm{THz}$, $1000 \mathrm{THz}$. Also, the transition from high reflectance to high absorptance is relatively sharp at $f_{c} \simeq 150 \mathrm{THz}$. 


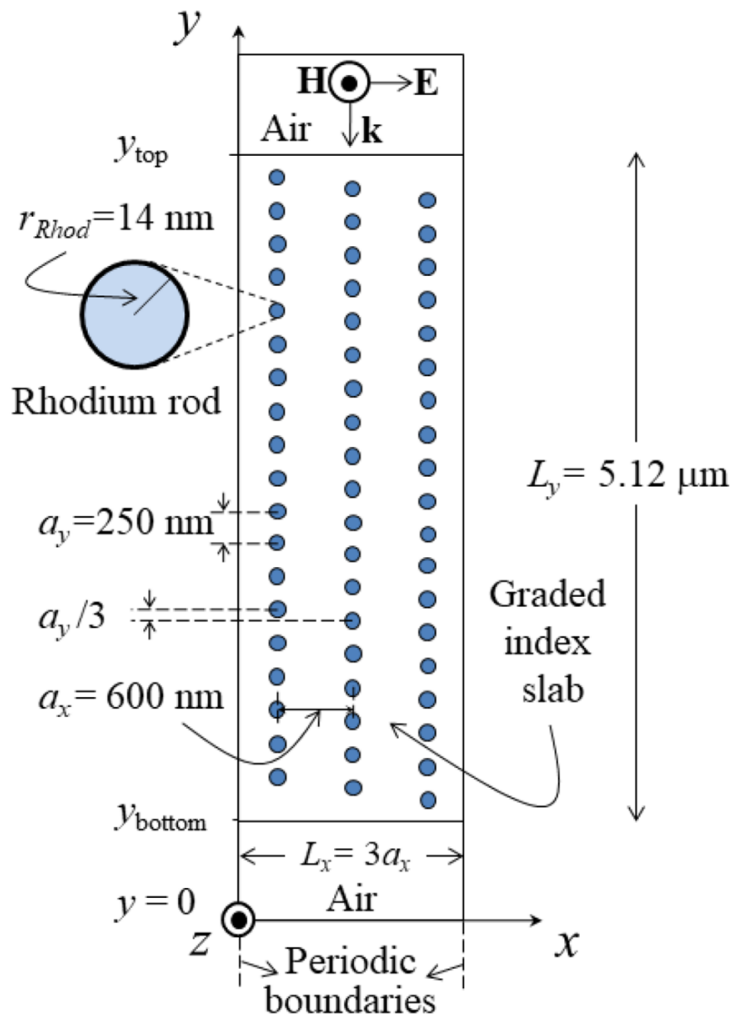

(a)

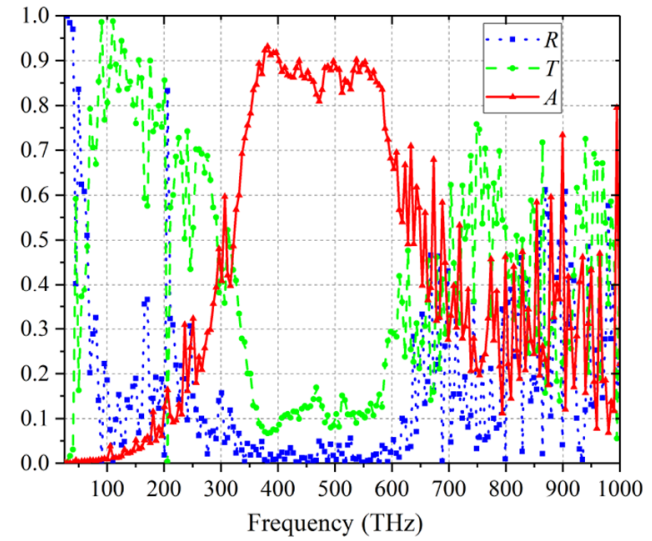

(b)

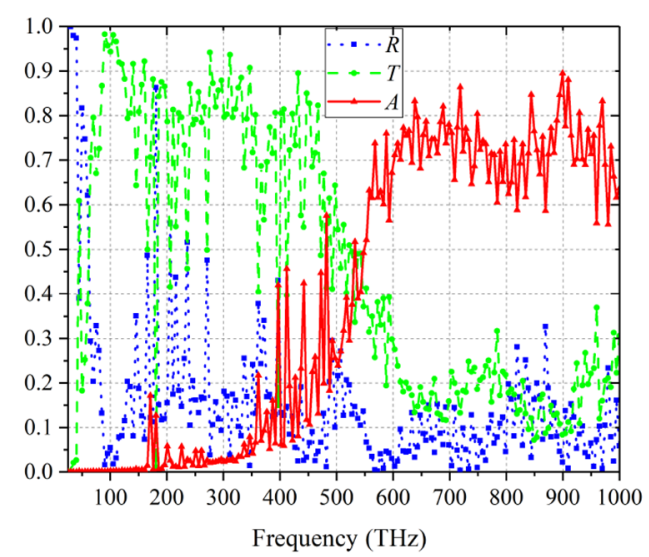

(c)

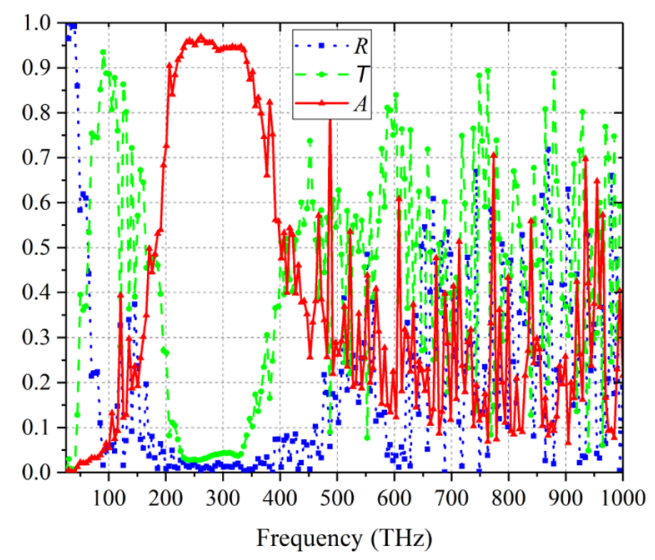

(d)

Fig. 3. (a) Schematic diagram (not according to scale) of the unit cell for a structure consisting of the graded index dielectric slab shown in Figure 2b, with three columns of rhodium rods embedded in it. Each rod has radius $r=14 \mathrm{~nm}$, and the rods are separated horizontally by $a_{x}=600 \mathrm{~nm}$ and vertically by $a_{y}=250 \mathrm{~nm}$. (b-d) Numerically calculated $R$, $T$, and $A$ spectra for TM normal incidence on the structure shown in (a), with metal rods consisting of: rhodium of radius $14 \mathrm{~nm}$ in (b), tungsten of radius $8 \mathrm{~nm}$ in (c), and TiN of radius $24 \mathrm{~nm}$ in $(\mathrm{d})$.

In Figure 4c-e, we explore how the proposed structure performs for TM light having oblique incidence. Figure 4c is a two-dimensional (2D) color contour plot for reflectance in terms of frequency and incidence angle, while Figure $4 \mathrm{~d}$ shows transmittance, and Figure 4e shows absorptance. We can see that for $f_{\text {inc }}<150 \mathrm{THz}$, reflectance is close to 1 (hence emittance is negligible and suppressed) for all values of the incidence angle. However, for $f_{\text {inc }}>200 \mathrm{THz}$, 


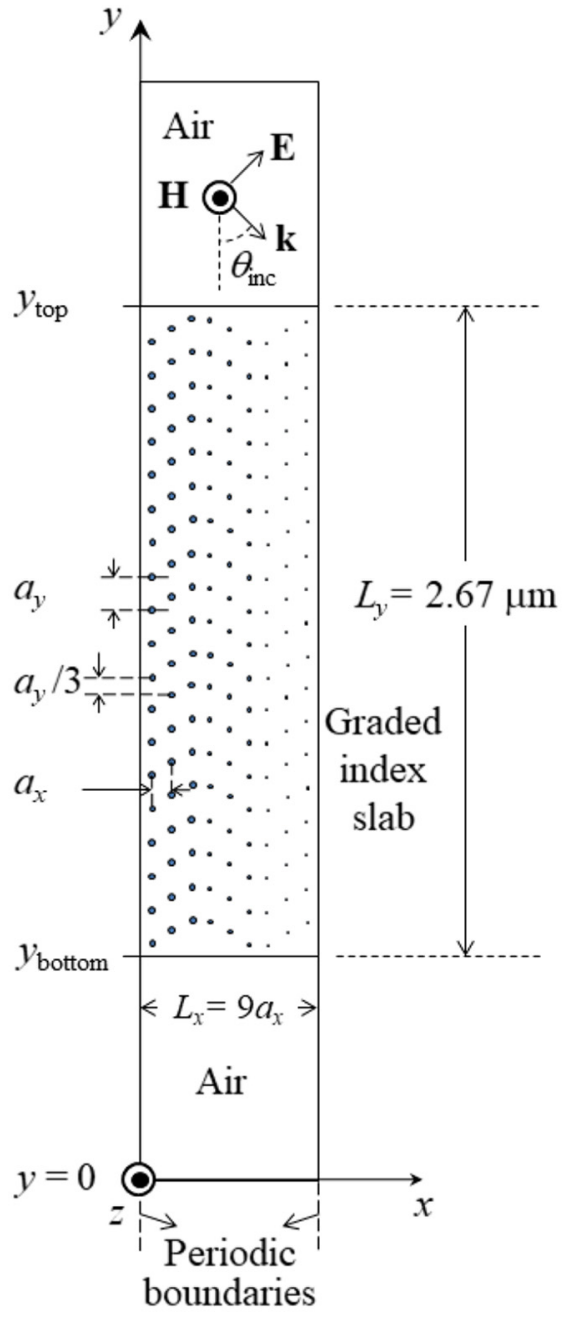

(a)

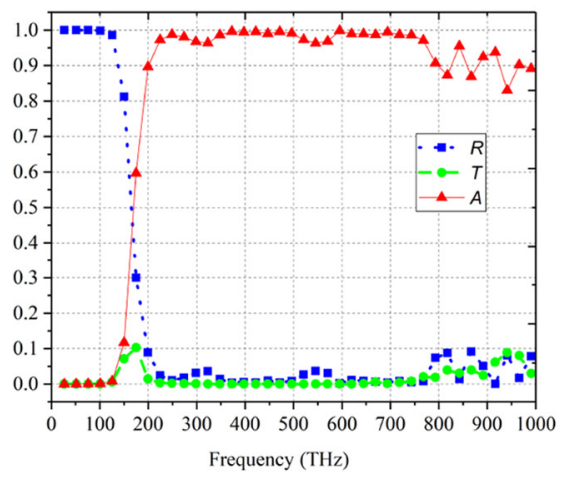

(b)

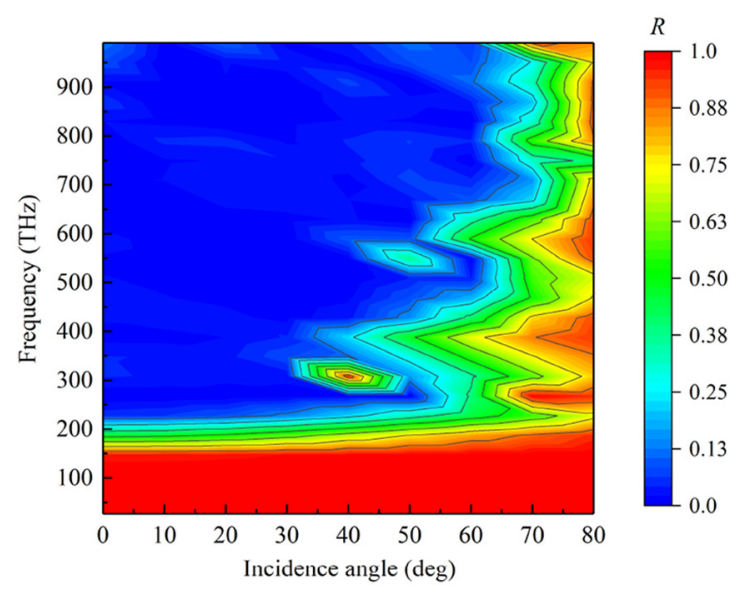

(c)

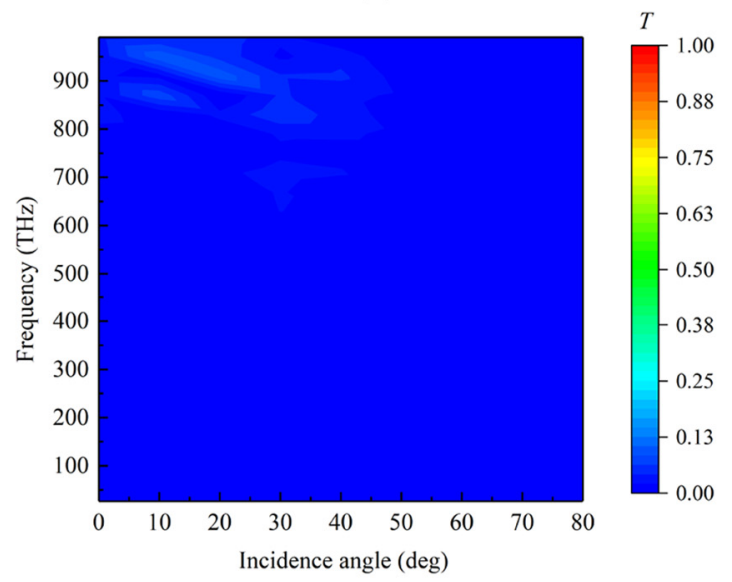

(d)

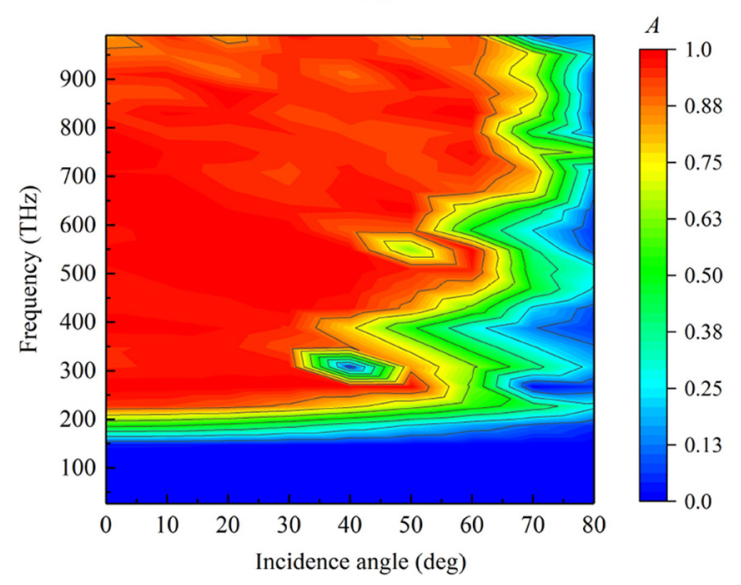

(e)

Fig. 4. (a) Schematic diagram showing TM polarized light incident at an angle $\theta_{\text {inc }}$ on a unit cell of the proposed selective absorber structure which consists of a graded index slab having thickness $L_{y}=2.67 \mu \mathrm{m}$, with nine columns and nineteen rows of metallic nanorods embedded in it. The three rod columns at left are made out of TiN and have a rod radius of $24 \mathrm{~nm}$. The three middle ones are made out of rhodium and have a rod radius of $14 \mathrm{~nm}$. The three rod columns at right are made out of TiN and have a rod radius of $8 \mathrm{~nm}$. The horizontal interparticle spacing is $a_{x}=800 \mathrm{~nm}$, and the vertical one is $a_{y}=130 \mathrm{~nm}$. Consecutive columns of the same metal type are vertically shifted by $a_{y} / 3$ to ensure that the rods sense a smooth variation in the slab's refractive index. (b) Numerically calculated $R, T$, and $A$ spectra for the structure shown in (a) in the case of normally incident $\left(\theta_{\text {inc }}=0\right)$ TM polarized light. (c-e) Two-dimensional (2D) color contour plots for $R, T$, and $A$, in terms of frequency and $\theta_{\text {inc }}$, for TM light incident on the structure shown in (a). 
absorptance is high over a significant angular range $0<\theta_{\text {inc }}<60^{\circ}$, enabling a benefit from concentrated solar thermal power conversion.

Although this proposed structure is designed to work for TM polarized incident light, one can, in principle, easily extend its functionality to both TE and TM polarizations, by replacing the nanowires with nanospheres, for which the Frohlich condition of SPR becomes $\operatorname{Re}\left(\varepsilon_{m}\right)=-2 \varepsilon_{d}[21]$. In this case, the numerical simulations would need to be carried out in three dimensions, and would therefore be a lot more demanding than the two-dimensional simulations for the nanorods.

Regarding the choice of radii, we limited them to 8-25 nm, since, for smaller radii, the metal dielectric function would have a dominant size dependance as well. As for the $25 \mathrm{~nm}$ upper limit, it was set in order to ensure that $r \ll \lambda$ over the very broad frequency range considered, so that the dominant SPR contribution is from the dipole mode, to which the Frohlich condition corresponds $[20,21]$. The specific radius choice for each metal type was also done so as the absorptance of the proposed structure is closest to the ideal targeted one.

Since the numerical simulation results did not reveal deviations from the design predictions based on the electric dipole resonance alone, we did not explore the possibility of induced magnetic resonances in our proposed design structure. If the structure has induced magnetic resonances at the same frequency as the electric dipolar ones, as in reference [33] for example, then this coincidence would also serve our objective of frequency selective absorption and tunability of thermal emission.

Although we assumed a lossless graded index material, both in the design and numerical validation of our proposed selective absorber, the loss effect needs to be investigated, given the wide frequency range considered and the high $T_{h}$ values. For example, for a pSi graded index material, absorption becomes negligible at $200 \mathrm{THz}$ $(0.8 \mathrm{eV})[34]$. Hence, the absorptive loss in such a graded index material should not affect our zero-absorptance objective below $150 \mathrm{THz}$, regardless of incidence angle. For frequencies above $200 \mathrm{THz}$, as the refractive index of $\mathrm{pSi}$ is decreased, the absorptive loss decreases and the frequency dependence of the real part of the dielectric function $(\operatorname{Re}[\varepsilon(\omega)])$ becomes less pronounced [24]. This frequency dependence of $\operatorname{Re}[\varepsilon(\omega)]$ is expected to broaden the SPRs, which is desired in our selective absorber design, noting that, for $\mathrm{pSi}, \operatorname{Re}[\varepsilon(\omega)]$ is positive over all the frequency range considered (up to $1000 \mathrm{THz}$ ) [24], which ensures that the Frohlich condition can be satisfied. As for angular dependence at frequencies above $200 \mathrm{THz}$, the loss in the graded index material is expected to increase with increasing incidence angle (longer path), and then drop to zero once grazing incidence is approached at $\theta_{\text {inc }}=90^{\circ}$.

If the graded index material has significant dispersion for frequencies above $200 \mathrm{THz}$, then this dispersion can be easily taken into consideration, since we are optimizing the structure's absorption separately in each of the three above-considered frequency intervals. Let us suppose for example that, due to material dispersion, the graded index $n(y)$ varies between 1.7 (at slab's top) and 2.5 (at slab's bottom) for frequencies between 600 and $1000 \mathrm{THz}$, while still having the $n(y): 1.2-2.2$ profile for frequencies less than $600 \mathrm{THz}$. Then, in this case, the W rods (responsible about absorption in the $600-1000 \mathrm{THz}$ interval) would need to be replaced by rods of a metal having $\omega_{p}$ such that $\left[\frac{\omega_{p}}{\sqrt{1+2.5^{2}}}, \frac{\omega_{p}}{\sqrt{1+1.7^{2}}}\right]$, which is $\left[0.37 \omega_{p}\right.$, $\left.0.5 \omega_{p}\right]$, covers the $600-1000 \mathrm{THz}$ interval.

\section{Flexible tunability of thermal emission based on proposed design approach}

In some applications, thermal emission needs to be designed with high sensitivity. Such a delicate control of emission requires limiting the broadening effect due to interparticle interactions, which can be achieved by increasing the interparticle separation. So, in order to manage the numerically demanding simulations in this case, we focus again on the TM polarization only and we consider a periodically repeating structure (along $x$ ), whose unit cell consists of a single column of Rh rods embedded in the graded index slab, such that the vertical interparticle separation is $a_{y}=250 \mathrm{~nm}$, and such that the unit cell size along $x$ is $a_{x}=600 \mathrm{~nm}$.

Our goal is to link this structure's absorptance over a certain frequency interval to the vertical positions $y$ of corresponding Rh rods in the graded index slab. So, we consider (in a separate simulation) a single Rh rod of radius $r_{\mathrm{Rh}}=14 \mathrm{~nm}$, and embed it in a homogeneous dielectric medium of uniform refractive index $n$, as shown in Figure 2a. By performing the same numerical simulations mentioned in Section 3, we calculate the absorptance $A(=1-R-T)$ in terms of frequency $f$ and surrounding refractive index $n$ for TM incident light. The result is shown as a $2 \mathrm{D}$ color contour plot in Figure $5 \mathrm{a}$, where we can see peak absorption for central frequencies varying between $380 \mathrm{THz}$ and $580 \mathrm{THz}$, matching the predictions of equation (6).

By using equation (5) for the refractive index spatial profile in the graded index slab, we then calculate the vertical position $y$ in the slab corresponding to each value of $n$, and in Figure 5b, we plot absorptance for the single rod case again, but in terms of frequency $f$ and relative vertical position $\left(y-y_{\text {bottom }}\right) / L_{y}$ in the graded index slab.

As an example on how to flexibly tune thermal emission, let us consider tuning the absorption in a specific frequency interval around $f=440 \mathrm{THz}$ in the abovementioned periodic structure whose unit cell consists of $19 \mathrm{Rh}$ rods vertically separated by $a_{y}=250 \mathrm{~nm}$, and embedded in the same graded index slab as before. The slab's thickness is $L_{y}=5.125 \mu \mathrm{m}$ in this case, and the horizontal size of the computational domain (unit cell) is $a_{x}=600 \mathrm{~nm}$. From Figure 5b, we predict that the absorption around $440 \mathrm{THz}$ is mainly due to the specific rods having $0.33 L_{y}+y_{\text {bottom }}<y<0.67 L_{y}+y_{\text {bottom. }}$. To validate this, we perform simulations again for TM light normally incident on the single-column structure, with periodic boundary conditions imposed on the unit cell's 


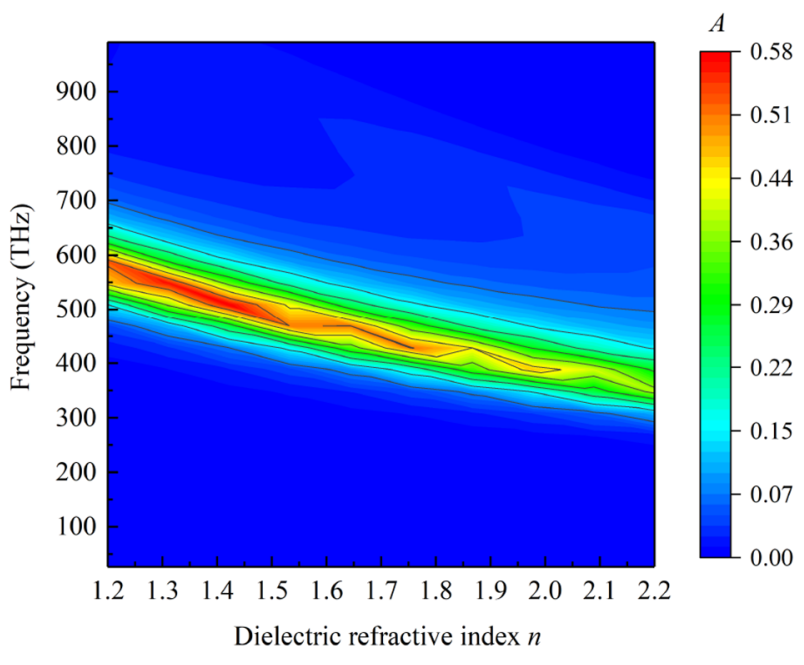

(a)

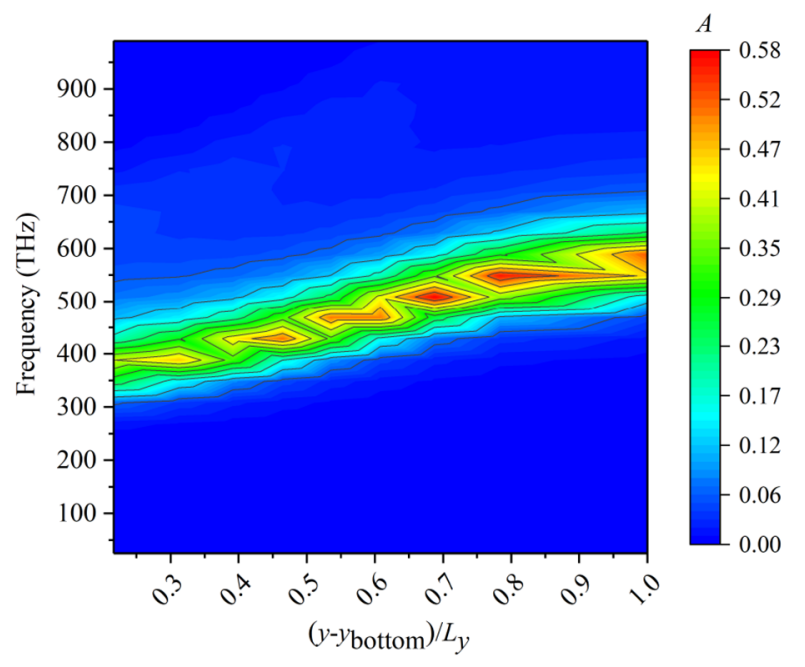

(b)

Fig. 5. (a) $2 \mathrm{D}$ color contour plot for the absorptance $(A)$ as a function of frequency $f$ and surrounding refractive index $n$, for the singlerod case shown in Figure 2a. (b) $2 \mathrm{D}$ color contour plot for the absorptance $(A)$ as a function of frequency $f$ and relative vertical position $\left(y-y_{\text {bottom }} / L_{y}\right)$ in the case where the single rhodium rod of radius $14 \mathrm{~nm}$ is placed at a vertical position $y$ in a graded index slab of

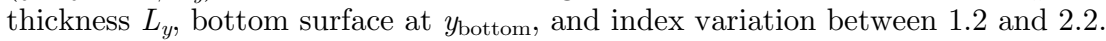

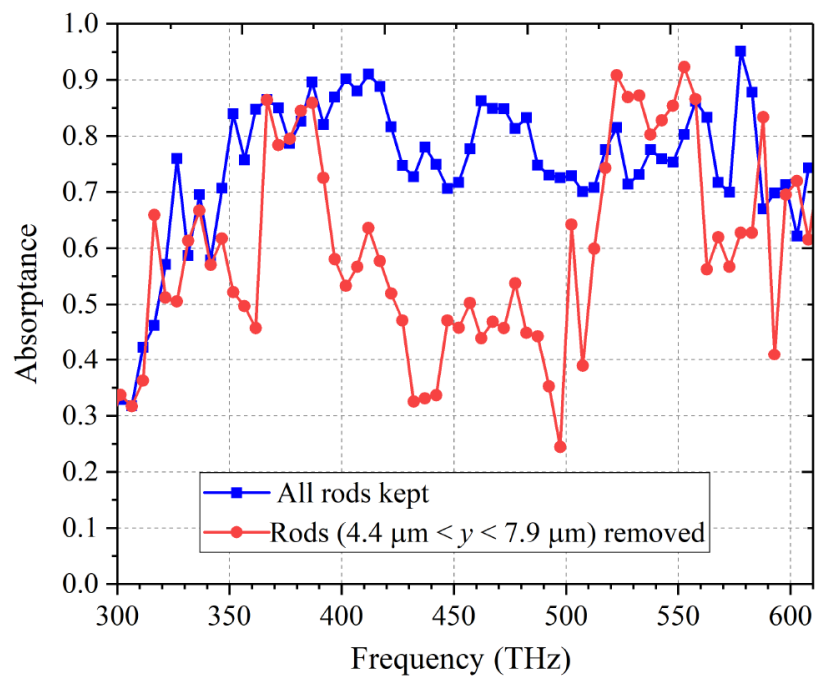

(a)

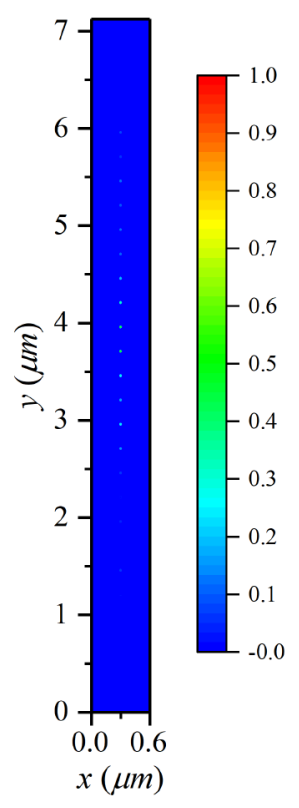

(b)

Fig. 6. (a) Absorptance spectrum (in blue) for a TM polarized light incident on a structure whose unit cell has size $a_{x}=600 \mathrm{~nm}$, and consists of a single column of $19 \mathrm{Rh}$ rods separated by $a_{y}=250 \mathrm{~nm}$. The red curve corresponds to the absorptance spectrum when the rods having $2.69 \mu \mathrm{m}<y<4.43 \mu \mathrm{m}$ are removed. (b) $2 \mathrm{D}$ color contour plot for the normalized volume density of power dissipation $(\mathbf{J} \cdot \mathbf{E} *)$ in the case of a TM polarized light of frequency $f=440 \mathrm{THz}$ incident on the structure mentioned in (a), before removing any rods.

sides at $x=0$ and $x=600 \mathrm{~nm}$. The absorptance spectrum is shown (in blue) in Figure 6a in this case, and in Figure 6b, we show a $2 \mathrm{D}$ color plot for the (normalized) volume density of power dissipation $\left(\mathbf{J} \cdot \mathbf{E}^{*}\right)$ when the incident light frequency is $440 \mathrm{THz}$. We can see in Figure 6b that, indeed, most of the rods with $0.33 L_{y}+$ $y_{\text {bottom }}<y<0.67 L_{y}+y_{\text {bottom }}$ (corresponding to $2.69 \mu \mathrm{m}<$ $y<4.4 \mu \mathrm{m}$ ) have the highest density of power dissipation, which confirms the prediction (based on Fig. 5b), that these particular rods are mainly responsible about absorption at 


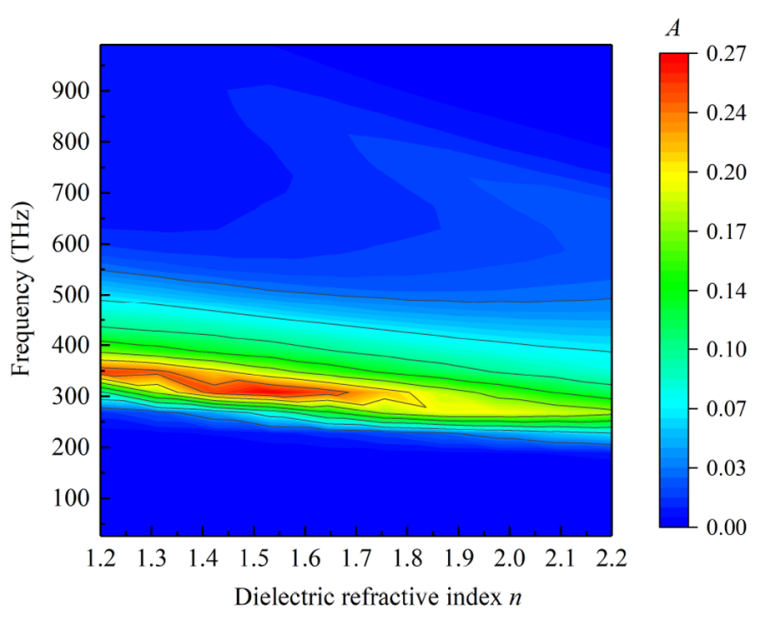

(a)

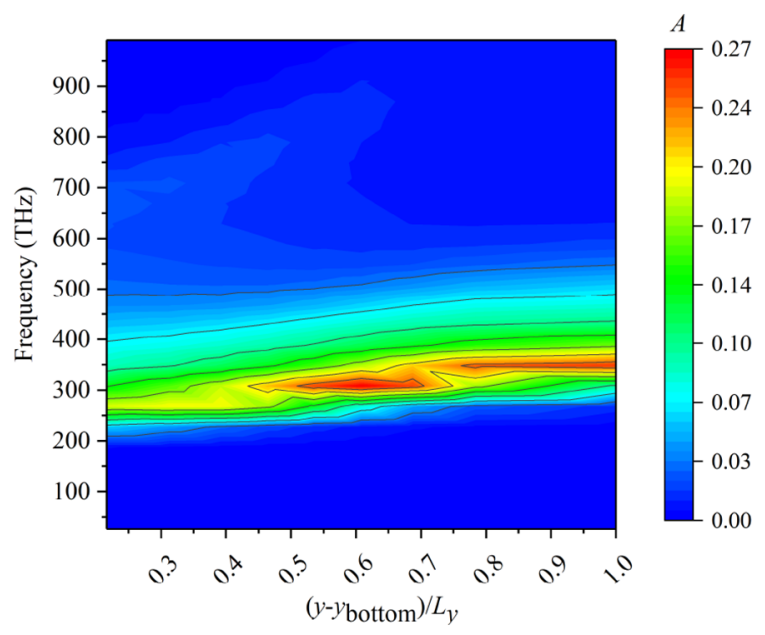

(c)

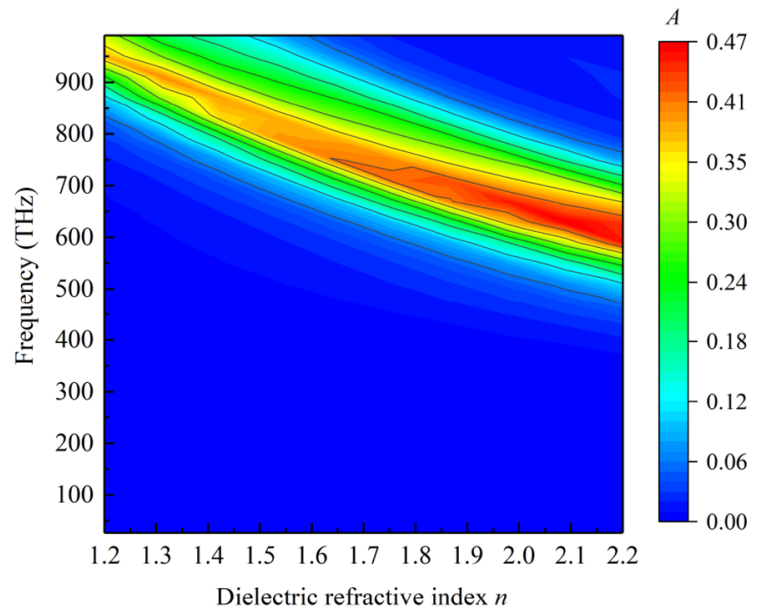

(b)

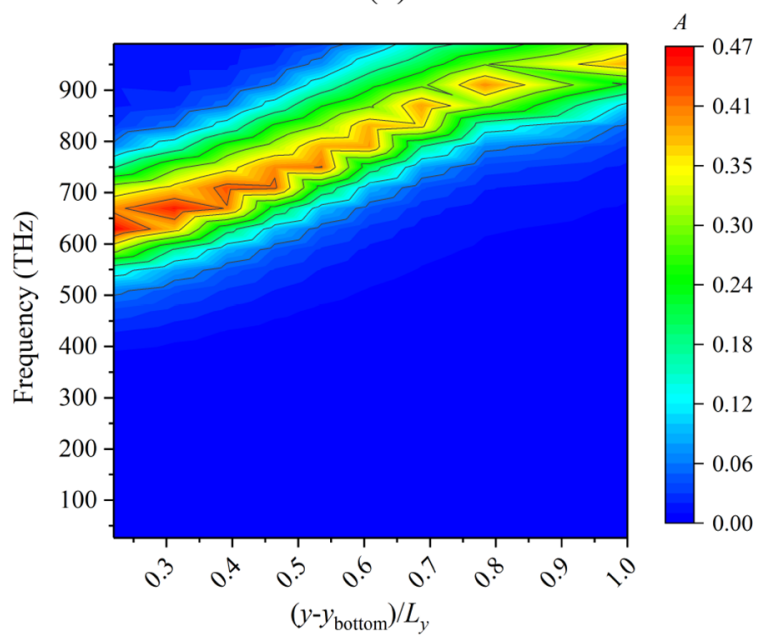

(d)

Fig. 7. 2D color contour plots of absorptance (similar to those in Fig. 5, but in the cases where the single metallic rod consists of: TiN (cases (a) and (c)) with radius of $24 \mathrm{~nm}$, and $\mathrm{W}$ (cases (b) and (d)) with radius of $8 \mathrm{~nm}$.

$440 \mathrm{THz}$. So, by keeping/removing these specific rods (at the design stage), one could, in principle, enhance/suppress absorption (and hence emission) at frequencies around $440 \mathrm{THz}$. In fact, as shown in in Figure 6a, there is a significant suppression in the absorptance spectrum around $440 \mathrm{THz}$, upon removal of the specific rods having $0.33 L_{y}+$ $y_{\text {bottom }}<y<0.67 L_{y}+y_{\text {bottom }}$.

This tunability of thermal emission is in fact a novel approach in the control of thermal emission, and is beneficial not just for solar energy harvesting, but it also enables many other technologies that could benefit from spectral control, such as sensing, camouflage, and other thermal management applications.

For completeness, we show in Figure $7(\mathrm{a}-\mathrm{d}), 2 \mathrm{D}$ color contour plots similar to those of Figure 5, but corresponding to TiN and $\mathrm{W}$ rods, respectively. These numerical results not only confirm the predicted frequency ranges for absorption, but also more importantly, they can be used to control TM thermal emission over the intervals [243 THz,
$346 \mathrm{THz}]$ and $[620 \mathrm{THz}, 1000 \mathrm{THz}]$. One should note, however, that in the TiN case, due to the low $\omega_{p}$, the frequency of peak absorption has a slower dependence on $n$ and $y$, and the emission control is expected to be a bit more difficult than the cases of Rh and W (since the multiple rods involved in absorption at a certain frequency, might also be involved in absorption at many other frequencies as well). This limitation can be overcome by using a dieletric profile with an index variation wider than the $1.2-2.2$ interval considered, which results in a steeper dependence of the absorption frequency on $y$, enabling a finer control over thermal emission even in the low frequency interval 243-346 THz.

Finally, depending on the specific application for which thermal emission design/control is needed, one could certainly consider using various other non-sinusoidal refractive index profiles for the dielectric slab, and also possibly wider index variations. Many such index profiles have been implemented and reported in the literature [35]. 
One should, however, pay special attention to the thermal, chemical, and mechanical stability considerations specific to the application of interest, which, for example, put a limitation on $T_{h}$ in the selective absorber design case. Also, from the practical side, the manipulation of nanosized rods to be embedded in the graded index slab will involve some technical difficulties.

\section{Conclusion}

In conclusion, we proposed and numerically validated a highly efficient solar selective absorber structure based on localized transverse SPRs in nanorods embedded in a graded index dielectric. The proposed structure features a TM reflectance of 1 at low IR frequencies $(f<150 \mathrm{THz})$, and a TM absorptance reaching $0.99-1$ over a wide portion of the solar spectrum above $200 \mathrm{THz}$. We also explained how the proposed structure can be modified so as to extend its selective absorber functionality to both TE and TM polarizations, and also to flexibly tune thermal emission in a certain frequency range (enhancing/suppressing it by keeping/removing rods from specific locations). Hence, our proposed strucure could benefit not only solar energy harvesting devices (solar heating, solar TPV, solar thermal CSP, etc.), but also has the promise to impact and advance other technologies requiring spectral control of thermal emission, including but not limited to sensing, camouflage, etc.

\section{References}

1. C.F. Guo, T. Sun, F. Cao et al., Metallic nanostructures for light trapping in energy-harvesting devices, Light Sci. Appl. 3, e161 (2014)

2. Y. Takeda, H. Iizuka, N. Yamada et al., Light trapping for photovoltaic cells using polarization-insensitive angle-selective filters under monochromatic illumination, Appl. Opt. 56, 5761 (2017)

3. S.V. Boriskina, H. Ghasemi, G. Chen, Plasmonic materials for energy: from physics to applications, Mater. Today 16, 10 (2013)

4. B.L. Sopori, J. Madjdpour, W. Chen, Y. Zhang, Lighttrapping in a-Si solar cells: a summary of the results from pv optics, April 27, 1999; Golden, Colorado (digital.library.unt. edu/ark: $67531 / \operatorname{metadc} 703664 / \mathrm{m1} / 3 /$ : accessed July 15, 2018), University of North Texas Libraries, Digital Library, digital.library.unt.edu; crediting UNT Libraries Government Documents Department

5. A. Marti, A. Luque, Next Generation Photovoltaics: High Efficiency through Full Spectrum Utilization, 1st edn. Series in Optics and Optoelectronics (CRC PRESS, Florida, 2003), Vol. 4

6. Q. Ni, H. Alshehri, Y. Yang et al., Plasmonic light trapping for enhanced light absorption in film-coupled ultrathin metamaterial thermophotovoltaic cells, Front. Energy 12, 185 (2018)

7. G.J. Snyder, Thermoelectric energy harvesting (Springer, Boston, MA, 2009)

8. H.J. Goldsmid, The physics of thermoelectric energy conversion, 2053-2571 (Morgan and Claypool Publishers, CA, 2017)
9. J. Yellowhair, C.K. Ho, J.D. Ortega, J.M. Christian, C.E. Andraka, Testing and optical modeling of novel concentrating solar receiver geometries to increase light trapping and effective solar absorptance, Proc. SPIE 9559, High and Low Concentrator Systems for Solar Energy Applications X, 95590A (5 September 2015); doi: 10.1117/12.2186647

10. C. Honsberg, S. Bowden, https://pveducation.org/ (accessed July 10, 2018)

11. C. Chang, C. Yang, Y. Liu et al., Efficient solar thermal energy harvest driven by interfacial plasmonic heating assisted evaporation, ACS Appl. Mater. Interfaces 8, 23412 (2016)

12. S. Ishii, R.P. Sugavaneshwar, T. Nagao, Titanium nitride nanoparticles as plasmonic solar heat transducers, J. Phys. Chem. C 120, 2343 (2016)

13. Y. He, M. Chen, X. Wang, Y. Hu, Plasmonic multi thorny gold nanostructures for enhanced solar thermal conversion, Sol. Energy 171, 73 (2018)

14. M. Chen, Y. He, Plasmonic nanostructures for broadband solar absorption based on the intrinsic absorption of metals, Sol. Energy Mater. Sol. Cells 188, 156 (2018)

15. J.C.C. Fan, S.A. Spura, Selective black absorbers using rf sputtered cr2o3/cr cermet films, Appl. Phys. Lett. 30, 511 (1977)

16. H.G. Craighead, R.A. Buhrman, Optical properties of selectively absorbing $\mathrm{Ni} / \mathrm{Al}_{2} \mathrm{O}_{3}$ composite films, Appl. Phys. Lett. 31, 423 (1977)

17. V. Teixeira, E. Sousa, M.F. Costa et al., Spectrally selective composite coatings of crcr2o3 and moal2o3 for solar energy applications, Thin Solid Films 392, 320 (2001)

18. K.D. Olson, J.J. Talghader, Absorption to reflection transition in selective solar coatings, Opt. Express 20, A554 (2012)

19. C. Kennedy, Review of mid- to high-temperature solar selective absorber materials, techrep., National Renewable Energy Laboratory, Golden, CO, USA (2002)

20. M.A. Garcia, Surface plasmons in metallic nanoparticles: fundamentals and applications, J. Phys. D: Appl. Phys. 44, 283001 (2011)

21. G.C. van de Hulst, Light scattering by small particles (Dover, New York, 2000)

22. S. Callard, A. Gagnaire, J. Joseph, Fabrication and characterization of graded refractive index silicon oxynitride thin films, J. Vac. Sci. Technol. A 15, 2088 (1997)

23. S. Ilyas, M. Gal, Graded refractive index devices from porous silicon, in: IEEE 2006 International Conference on Nanoscience and Nanotehnology, Brisbane, QLD, Australia, 2006

24. G. Korotcenkov, Porous silicon: from formation to application (CRC Press, Taylor and Francis Group, New York, 2016), Vol. 1

25. R. Herino, A. Perio, K. Barla et al., Microstructure of porous silicon and its evolution with temperature, Mater. Lett. 2, 519 (1984)

26. V.A. Labunov, V.P. Bondarenko, V.E. Borisenko et al., High temperature treatment of porous silicon, Phys. Status Solidi A 102, 193 (1987)

27. S.M. Weiss, M. Molinari, P.M. Fauchet, Temperature stability for silicon based photonic bandgap structures, Appl. Phys. Lett. 83, 1980 (2003)

28. E.D. Palik, E.J. Prucha, Handbook of optical constants of solids (Academic Press, Boston, MA, 1997)

29. Y. Gutierrez, R. Alcaraz de la Osa, D. Ortiz, et al., Plasmonics in the ultraviolet with aluminum, gallium, magnesium and rhodium, Appl. Sci. 8, 64 (2018) 
30. C. Bohren, D.R. Huffman, Absorption and scattering of light by small particles, Wiley Science Paperback Series (Wiley VCH, Weinheim, 1998)

31. N.W. Ashcroft, N.D. Mermin, Solid state physics (Cengage Learning, New York, 1976)

32. P. Patsalas, N. Kalfagiannis, S. Kassavetis, Optical properties and plasmonic performance of titanium nitride, Materials 8, 3128 (2015)
33. A. Alu, A. Salandrino, N. Engheta, Negative effective permeability and left handed materials at optical frequencies, Opt. Express 14, 1557 (2006)

34. D.J. Lockwood, G.C. Aers, L.B. Allard et al., Optical properties of porous silicon, Can. J. Phys. 70, 1184 (1992)

35. N. Ishikura, M. Fujii, K. Nishida et al., Broadband rugate filters based on porous silicon, Opt. Mater. 31, 102 (2008)

Cite this article as: Rafif Hamam, Ali J. Sabbah, Highly efficient and tunable selective absorber design based on metallic nanoparticles in a graded index dielectric, EPJ Appl. Metamat. 6, 7 (2019) 